\title{
SYMMETRIES AND CONSERVATION LAWS OF 2-DIMENSIONAL IDEAL PLASTICITY
}

\author{
by S. I. SENASHOV and A. M. VINOGRADOV
}

(Received 27th January 1987)

\section{Introduction}

Symmetry theory is of fundamental importance in studying systems of partial differential equations. At present algebras of classical infinitesimal symmetry transformations are known for many equations of continuum mechanics $[1,2,4]$. Methods for finding these algebras go back to $\mathrm{S}$. Lie's works written about 100 years ago. In particular, knowledge of symmetry algebras makes it possible to construct effectively wide classes of exact solutions for equations under consideration and via Noether's theorem to find conservation laws for Euler-Lagrange equations. The natural development of Lie's theory is the theory of "higher" symmetries and conservation laws [5].

The most significant aspect of it is the possibility of calculating explicitly all conservation laws for arbitrary systems of differential equations, in particular for those for which the Noether theorem is not applicable.

It seems that the simplest approach to the theory of higher symmetries and conservation laws is in the language of generating functions (see below). These are functions of independent and dependent variables and their derivatives of any order as well. From this point of view the classical theory [4] appears to be a special case of the "higher theory", namely, the case when generating functions depend only on derivatives of order $\leqq 1$ and, moreover, satisfy some additional conditions.

Our purpose in this paper is to find all higher symmetries and conservation laws for equations of the plane static ideal plasticity problem. The remarkable fact is that this problem admits an infinite algebra of symmetries and an infinite group of conservation laws. Exact formulations are contained in Theorems 1-4.

It was not our aim in this paper to apply the results obtained. This will be done elsewhere. Also we would like to remark that this paper demonstrates how the general theory presented in [5] works in concrete problems. An interested reader may consult the book [3] for both technical and conceptual details of the theory.

\section{Preliminary}

Let $x=\left(x_{1}, \ldots, x_{n}\right)$ be independent variables and $u=\left(u^{1}, \ldots, u^{m}\right)$ be dependent ones. Suppose that the system of differential equations under consideration has the form

$$
F=0 \text {, }
$$


where $F=\left(F_{1}, \ldots, F_{r}\right), F_{i}=F_{i}\left(x, u, \ldots, u_{(s)}\right)$ and $u_{(l)}$ is the totality of all partial derivatives of order $l$ of functions $u^{i}$ with respect to variables $x_{j}$.

1. Roughly speaking an evolution system

$$
u_{\tau}^{i}=f^{i}\left(x, u, u_{(1)}, \ldots, u_{(s)}\right), \quad u_{\tau}^{i}=\frac{\partial u^{i}}{\partial \tau}
$$

where $\tau$ is a new independent variables, can informally be considered as a higher symmetry of the system $(0.1)$ if $u(x, \tau)$ is its solution for every fixed $\tau$ provided that $u(x, 0)$ is and also some suitable boundary conditions are fulfilled. We will not give here the exact definitions (see [3], [5]). Instead we will describe how to find the functions $f=\left(f^{1}, \ldots, f^{m}\right)$ which are called generating functions of the higher symmetry $(0.2)$.

2. Consider the infinite-dimensional space $J^{\infty}$ with coordinates $x, u, p_{\sigma}^{i}$, where the symbol $p_{\sigma}^{i}$ corresponds to the derivative

$$
\frac{\partial^{|\sigma|} u^{i}}{\partial x_{1}^{i_{1}} \ldots \partial x_{n}^{i_{n}}}, \quad|\sigma|=i_{1}+\cdots+i_{n}, \quad \sigma=\left(i_{1}, \ldots, i_{n}\right)
$$

By a smooth function on $J^{\infty}$ we mean a smooth function in a finite number of coordinates on $J^{\infty}$. The algebra of all such functions will be denoted by $\mathscr{F}$.

The total derivative operator with respect to $x_{j}$ is

$$
D_{j}=\frac{\partial}{\partial x_{j}}+\sum_{\sigma, i} P_{\sigma j}^{i} \frac{\partial}{\partial p_{o}^{i}}
$$

where $\sigma j=\left(i_{1}, \ldots, i_{j}+1, \ldots, i_{n}\right)$, if $\sigma=\left(i_{1}, \ldots, i_{n}\right)$.

Let $D_{\sigma}=D_{1}^{i_{1}} \cdots \cdots \circ D_{n}^{i_{n}}$, if $\sigma$ is as above. The universal linearization operator for the system $(0.1)$ is defined as

$$
l_{F}=\left|\begin{array}{ccc}
\sum_{\sigma} \frac{\partial F_{1}}{\partial p_{\sigma}^{1}} D_{\sigma} & \cdots & \sum_{\sigma} \frac{\partial F_{1}}{\partial p_{\sigma}^{m}} D_{\sigma} \\
\vdots & & \vdots \\
\sum_{\sigma} \frac{\partial F_{r}}{\partial p_{\sigma}^{1}} D_{\sigma} & \cdots & \sum_{\sigma} \frac{\partial F_{r}}{\partial p_{\sigma}^{m}} D_{\sigma}
\end{array}\right| .
$$

This is a matrix differential operator. The system (0.1) defines the submanifold $\mathscr{Y}_{\infty}$ in $J^{\infty}$ which is given by means of the following infinite system of equations:

$$
D_{\sigma}\left(F_{i}\right)=0 \quad \forall \sigma, i .
$$

Via the system (0.3) a part of the coordinate functions $p_{\sigma}^{i}$ on $\mathscr{Y}_{\infty}$ can be expressed through the others which will be supposed functionally independent. The latter class of 
coordinates will be called internal ones (on $\mathscr{\mathscr { Y }}_{\infty}$ ) while the first will be called external ones. Of course, decomposition of variables into internal and external ones may be made by many different ways.

3. A function $g$ on $J^{\infty}$ may be restricted on $\mathscr{Y}_{\infty}$. To perform it external variables are to be replaced by suitable expressions of internal variables. The restriction of $g$ on $\mathscr{Y}_{\infty}$ is denoted by $\bar{g}$. Similarly the restriction $\bar{D}_{j}$ of the operator $D_{j}$ on $\mathscr{Y}_{\infty}$ is defined by the formula

$$
\bar{D}_{j}=\frac{\partial}{\partial x_{j}}+\tilde{\sum} \vec{p}_{\sigma j}^{i} \frac{\partial}{\partial p_{\sigma}^{i}}
$$

where "wave" means that summing is only by the internal variables $p_{\sigma}^{i}$.

Let also

$$
\begin{aligned}
& \bar{D}_{\sigma}=\bar{D}_{1}^{i_{1}} \circ \cdots \circ \bar{D}_{n}^{i_{n}}, \quad \sigma=\left(i_{1}, \ldots, i_{n}\right) \\
& l_{F}=\left|\begin{array}{ccc}
\tilde{\sum} \frac{\partial F_{1}}{\partial p_{\sigma}^{1}} \bar{D}_{\sigma} & \ldots & \tilde{\sum} \frac{\partial F_{1}}{\partial p_{\sigma}^{m}} \bar{D}_{\sigma} \\
\vdots & & \vdots \\
\tilde{\sum} \frac{\partial F_{r}}{\partial p_{\sigma}^{1}} \bar{D}_{\sigma} & \ldots & \tilde{\sum} \frac{\partial F_{r}}{\partial p_{\sigma}^{m}} \bar{D}_{\sigma}
\end{array}\right| .
\end{aligned}
$$

4. We need the following result (see [3], [5]): (0.2) is a symmetry of the system (0.1) if and only if

$$
\Gamma_{F}(\bar{f})=0 \text {. }
$$

Here $f$ is understood as a column-vector. It should be noted that two symmetries with generating functions $f$ and $g$ are the same if $\bar{f}=\bar{g}$.

We associate with every generating function $f$ the operator

$$
\ni_{f}=\sum_{\sigma, i} D_{\sigma}\left(f_{i}\right) \frac{\partial}{\partial p_{\sigma}^{i}}
$$

on $J$ which is called an evolutionary derivation operator. The totality of all generating functions on $J^{\infty}$ forms a Lia algebra with respect to the "higher Jacobi bracket":

$$
\{f, g\}=\ni_{f}(g)-\ni_{g}(f) .
$$

In its turn the totality sym $\mathscr{Y}$ of all higher symmetries of the system $(0.1)$ is a Lie algebra with respect to the higher Jacobi bracket restricted to $\mathscr{Y}_{\infty}$.

5. The classical symmetry theory imbeds into the "higher" one as follows. Let

$$
X=\sum_{i} a_{i} \frac{\partial}{\partial x_{i}}+\sum_{j} b_{j} \frac{\partial}{\partial u_{j}}, \quad a_{i}=a_{i}(x, u), \quad b_{j}=b_{j}(x, u)
$$


be an infinitesimal transformation of independent and dependent variables and

$$
U^{i}=d u^{i}-\sum_{j} p_{j}^{i} d x_{j}
$$

Then the generating function $f=\left(f^{1}, \ldots, f^{m}\right)$ corresponding to it is defined by

$$
\left.f^{i}=X\right\lrcorner U^{i}=b^{i}-\sum_{j} p_{j}^{i} a_{j}
$$

One can see that $X$ is determined completely by this function.

6. A $n$-vector $w=\left(w_{1}, \ldots, w_{n}\right), w_{i}=w_{i}\left(x, u, \ldots, u_{(s)}\right)$ is called a conserved current for the system (0.1) if

$$
\operatorname{div} w=0 \quad \text { on } \mathscr{Y}_{\infty} .
$$

The latter means that $\operatorname{div} w=\sum_{\sigma, i} a_{\sigma}^{i} D_{\sigma}\left(F_{i}\right)$, or, equivalently, that $\operatorname{div} w=\sum A_{i}\left(F_{i}\right)$, where $A_{i}=\sum a_{\sigma}^{i} D_{\sigma}$ are some differential operators on $J^{\infty}$.

Let

$$
A_{i}^{*}=\sum_{\sigma}(-1)^{|\sigma|} D_{\sigma} \circ a_{\sigma}^{i}
$$

Vector-function $V=\left(V_{1}, \ldots, V_{m}\right), \quad V_{i}=\overline{A_{i}^{*}(1)}$, is called generating for the current $w$. Conversely, it may be proved assuming some regularity conditions on (0.1) that every conserved current is determined by the associated generating function uniquely up to an unessential summand of the form rot $\Omega$ (see [5]). Two conserved currents are said to be equivalent if their difference has the form rot $\Omega$. A class of equivalent conserved currents is said to be a conservation law for $(0.1)$. The reader will find motivation of these definitions in [5].

7. From above we see that for "good" equations conservation laws are determined uniquely by their generating functions. By these we mean generating functions of the corresponding conserved currents. Therefore the problem of finding conservation laws reduces to finding generating functions.

The following result (see [5]) is central in solving the last problem: the generating function $\phi$ of a conservation law satisfies the equation

$$
\overline{l_{F}^{*}}(\bar{\phi})=0
$$

where the matrix operator $\overline{l_{F}^{*}}$ is formally conjugate to $T_{F}$.

Remember that the matrix differential operator $\Delta^{*}$ formally conjugated to a matrix operator $\Delta=\left\|\Delta_{i j}\right\|$ has as its entries scalar operators

$$
\left(\Delta^{*}\right)_{i j}=\left(\Delta_{j i}\right)^{*}
$$


and if $A=\sum_{\sigma} a_{\sigma} D_{\sigma}$ is a scalar operator then

$$
A^{*}=\sum_{\sigma}(-1)^{|\sigma|} D_{\sigma} \circ a_{\sigma} .
$$

8. Note that not every solution $\phi$ of the system $(0.5)$ is the generating function a conservation law. In order to be so it is necessary and sufficient that the following representation takes place

$$
I_{\phi}+\bar{A}^{*}=\bar{B} \circ I_{F},
$$

where the operator $A$ satisfies the equality

$$
A(F)=l_{F}^{*}(\phi) \quad \text { and } B=B^{*} \text { (see [5]). }
$$

9. Conservation laws can be generated by symmetries. Namely, let $f$ be the generating function of a symmetry and $g$ the generating function of a conservation law. Then the "function" $f[g]$, defined by

$$
f[g]=l_{g}(f)+\Delta^{*}(g)
$$

is the generating function of a certain conservation law. Here the operator $\Delta=\sum c_{r} D_{r}$, $c_{\tau} \in \mathscr{T}$, satisfies the equality (see [5]):

$$
l_{F}(f)=\Delta(F) .
$$

\section{Linearization of plasticity equations with von Mises condition}

Consider the system of differential equations describing the plane strained state of the medium with von Mises condition

$$
\begin{aligned}
& \sigma_{x}-2 k\left(\theta_{x} \cos 2 \theta+\theta_{y} \sin 2 \theta\right)=0 \\
& \sigma_{y}-2 k\left(\theta_{x} \sin 2 \theta-\theta_{y} \cos 2 \theta\right)=0,
\end{aligned}
$$

where $\sigma$ is the pressure, $\theta$ is the angle between the $x$-axis and the first main direction of the stress tensor, $k$ is the plasticity constant and a subscript denotes the corresponding derivative.

1. It is known [1] that the system (1.1) admits the following algebra $L_{5}$ of infinitesimal symmetry transformations generated by the operators:

$$
\begin{gathered}
X_{1}=\frac{\partial}{\partial x}, \quad X_{2}=\frac{\partial}{\partial y}, \quad X_{3}=x \frac{\partial}{\partial x}+y \frac{\partial}{\partial y}, \\
X_{4}=x \frac{\partial}{\partial y}-y \frac{\partial}{\partial x}+\frac{\partial}{\partial \theta}, \quad X_{5}=\frac{\partial}{\partial \sigma} .
\end{gathered}
$$


Corresponding generating functions (see (0.4)) are

$$
\begin{gathered}
f_{1}=\left(\begin{array}{c}
-\sigma_{x} \\
-\theta_{x}
\end{array}\right), \quad f_{2}=\left(\begin{array}{c}
-\sigma_{y} \\
-\theta_{y}
\end{array}\right), \\
f_{3}=\left(\begin{array}{c}
-x \sigma_{x}-y \sigma_{y} \\
-x \theta_{x}-y \theta_{y}
\end{array}\right), \quad f_{4}=\left(\begin{array}{c}
-y \sigma_{x}+x \sigma_{y} \\
-y \theta_{x}+x \theta_{y}+1
\end{array}\right), \\
f_{5}=\left(\begin{array}{l}
1 \\
0
\end{array}\right),
\end{gathered}
$$

where

$$
\begin{aligned}
& U^{1}=d \sigma-\sigma_{x} d x-\sigma_{y} d y \\
& U^{2}=d \theta-\theta_{x} d x-\theta_{y} d y
\end{aligned}
$$

Here $\sigma_{x}$ stands for $p_{1}^{1}, \sigma_{y}$ for $p_{2}^{1}$ etc.

In [1] solutions of the system (0.1) invariant with respect to $L_{5}$ are constructed.

2. It will be useful for our aims to transform the equation (1.1). First of all, let us introduce new dependent variables $\xi, \eta$ by

$$
\sigma=k(\xi+\eta), \quad \theta=\frac{1}{2}(\eta-\xi) .
$$

In these variables the system (1.1) takes the form

$$
\frac{\partial \xi}{\partial x}+\frac{\partial \xi}{\partial y} \operatorname{tg} \theta=0, \quad \frac{\partial \eta}{\partial x}-\frac{\partial \eta}{\partial y} \operatorname{ctg} \theta=0
$$

Secondly, let us interchange dependent and independent variables in the last system, i.e. put

$$
x=x(\xi, \eta), \quad y=y(\xi, \eta) .
$$

This leads under the condition $(D(x, y)) /(D(\xi, \eta)) \neq 0$ to the system

$$
\frac{\partial y}{\partial \eta}-\frac{\partial x}{\partial \eta} \operatorname{tg} \theta=0, \frac{\partial y}{\partial \xi}+\frac{\partial x}{\partial \xi} \operatorname{ctg} \theta=0 .
$$

Finally passing in (1.3) to the new dependent variables $\bar{x}, \bar{y}$ by means of

$$
x=\bar{x} \cos \theta-\bar{y} \sin \theta, \quad y=\bar{x} \sin \theta+\bar{y} \cos \theta
$$

we obtain the desired system

$$
\frac{\partial \bar{x}}{\partial \xi}+\frac{1}{2} \cdot \bar{y}=0, \quad \frac{\partial \bar{y}}{\partial \eta}+\frac{1}{2} \cdot \bar{x}=0 .
$$


To simplify notation the last system will be written in new variables as

$$
\frac{\partial u}{\partial x}+\frac{1}{2} \cdot v=0, \quad \frac{\partial v}{\partial y}+\frac{1}{2} \cdot u=0 .
$$

Below we study the system (1.5) which is denoted by $\mathscr{Y}$. We will find all higher symmetries and conservation laws for it. After performing the back transformation we will obtain symmetries and conservation laws for the initial system (1.1).

\section{Higher symmetries of the linearized equations (1.5)}

The universal linearization operator for the system is (see Section 0 )

$$
l_{F}=\left(\begin{array}{cc}
D_{x} & \frac{1}{2} \\
\frac{1}{2} & D_{y}
\end{array}\right), \quad D_{x}=D_{1}, D_{y}=D_{2}
$$

Therefore we have to solve the equation

$$
I_{F}(\bar{f})=0,
$$

where

$$
l_{F}=\left(\begin{array}{cc}
\bar{D}_{x} & \frac{1}{2} \\
\frac{1}{2} & \bar{D}_{y}
\end{array}\right)
$$

in order to find symmetries of (1.5) (see Section 0.4).

We will put $u_{(k, l)}=p_{(k, l)}^{1}, v_{(k, l)}=p_{(k, l)}^{2}$ for the multi-index $(k, l)$ and will choose variables $x, y$ and $u_{(k)}=u_{(0, k)}, v_{(k)}=v_{(k, 0)}$ as internal coordinates on $\mathscr{Y}_{\infty}$. We write $f \in \mathscr{F}_{k}$, if $f=$ $f\left(x, y, u, v, u_{(1)}, v_{(1)}, \ldots, u_{(k)}, v_{(k)}\right)$.

In this notation the operators $\bar{D}_{x}$ and $\bar{D}_{y}$ are written as follows:

$$
\begin{aligned}
& \bar{D}_{x}=\frac{\partial}{\partial x}+\frac{1}{2} \cdot v \frac{\partial}{\partial u}-v_{(1)} \frac{\partial}{\partial v}+\cdots+\frac{1}{4} \cdot u_{(n-1)} \frac{\partial}{\partial u_{(n)}}+v_{(n+1)} \frac{\partial}{\partial v_{(n)}}+\cdots \\
& \bar{D}_{y}=\frac{\partial}{\partial y}+u_{(1)} \frac{\partial}{\partial u}-\frac{1}{2} \cdot u \frac{\partial}{\partial v}+\cdots+u_{(n+1)} \frac{\partial}{\partial u_{(n)}}+\frac{1}{4} \cdot v_{(n-1)} \frac{\partial}{\partial v_{(n)}}+\cdots
\end{aligned}
$$

1. Classical symmetries of the equation (1.5). In this case the generating functions $f=(\phi \psi)$ depend on variables $x, y, u, v, u_{x}, v_{x}, u_{y}, v_{y}$ and therefore $f$ depends only on $x$, $y, u, v, u_{y}=u_{(1)}, v_{x}=v_{(1)}$. It is not difficult to see that the left hand side of the first of the equations (2.1) is a first order polynomial in the variable $v_{(2)}$. Therefore (2.1) holds iff coefficients of that polynomial are equal to zero. So $\left(\partial \Phi / \partial v_{(1)}\right)=0$ as being the coefficient of $v_{(2)}$. Similarly from the second of the equations $(2.1)$ we find $\left(\partial \psi / \partial u_{(1)}\right)=0$. Therefore taking into consideration that $\psi=-2 \bar{D}_{x} \phi, \phi=-2 \bar{D}_{y} \psi$ we see that

$$
\phi=A_{1} u_{(1)}+B_{1}, \quad \psi=A_{2} v_{(1)}+B_{2}, \quad A_{i}, B_{i} \in \mathscr{F}_{0} .
$$


Putting these expressions into (2.1) we see that its left-hand side is a linear polynomial with respect to the variables $u_{(1)}, v_{(1)}$. By the same reasons as above coefficients of $u_{(1)}, v_{(1)}$ are to be zero. The $u_{(1)}$-coefficient in the first of the equations $(2.1)$ is $D_{x} A_{1}$ and one finds easily from the equation $\bar{D}_{x} A_{1}=0$ that $A_{1}=A_{1}(y)$. The $v_{(1)}$-coefficient in the second of the equations (2.1) equals $\bar{D}_{y} A_{2}$ as well and $A_{2}=A_{2}(x)$. Similarly, consideration of other coefficients leads to

$$
\begin{aligned}
& B_{1}=-\frac{1}{2} \cdot A_{2} v+C_{1}, \\
& B_{2}=-\frac{1}{2} \cdot A_{1} u+C_{2} .
\end{aligned}
$$

Having this in mind one may conclude that $T_{F}(f)$ is a polynomial in $u$ and $v$. Finally, performing one similar step more we obtain

$$
\begin{aligned}
& \bar{\phi}=(\alpha y+\beta) u_{(1)}+(\alpha+\delta) u-\frac{1}{2} \cdot(-\alpha x+\gamma) v+h_{1}(x, y) \\
& \bar{\psi}=(-\alpha x+\gamma) v_{(1)}+\delta v-\frac{1}{2} \cdot(\alpha y+\beta) u+h_{2}(x, y),
\end{aligned}
$$

where $\alpha, \beta, \gamma, \delta$ are arbitrary constants and $\left(h_{1}, h_{2}\right)$ is an arbitrary solution of (1.5). It follows from (2.2) that the elements

$$
\begin{gathered}
S_{1}=\left(\begin{array}{c}
y u_{(1)}+\frac{1}{2} \cdot u+\frac{1}{2} \cdot x v \\
-x v_{(1)}-\frac{1}{2} \cdot v-\frac{1}{2} \cdot y u
\end{array}\right), \quad S_{0}=\left(\begin{array}{l}
u \\
v
\end{array}\right), \\
f_{1}^{0}=\left(\begin{array}{c}
u_{(1)} \\
-\frac{1}{2} \cdot u
\end{array}\right), \quad g_{1}^{0}=\left(\begin{array}{c}
-\frac{1}{2} \cdot v \\
v_{(1)}
\end{array}\right), \quad H=\left(\begin{array}{l}
h_{1} \\
h_{2}
\end{array}\right),
\end{gathered}
$$

generate additively the classical symmetry Lie algebra of (1.5) and

$$
\begin{gathered}
\left\{S_{1}, f_{1}^{0}\right\}=f_{1}^{0}, \quad\left\{S_{1}, g_{1}^{0}\right\}=g_{1}^{0}, \\
\left\{S_{0}, f_{1}^{0}\right\}=\left\{S_{0}, g_{1}^{0}\right\}=0, \quad\left\{S_{0}, H\right\}=-H, \\
\left\{f_{1}^{0}, H\right\}=\left(\begin{array}{c}
\frac{\partial h_{1}}{\partial y} \\
-\frac{1}{2} \cdot h_{1}
\end{array}\right), \quad\left\{g_{1}^{0}, H\right\}=\left(\begin{array}{c}
-\frac{1}{2} \cdot h_{2} \\
\frac{\partial h_{2}}{\partial x}
\end{array}\right), \\
\left\{S_{1}, H\right\}=\left(\begin{array}{c}
y \frac{\partial h_{1}}{\partial y}+\frac{1}{2} \cdot h_{1}+\frac{1}{2} \cdot x h_{2} \\
-\frac{1}{2} \cdot y h_{1}-x \frac{\partial h_{2}}{\partial x}-\frac{1}{2} \cdot h_{2}
\end{array}\right) .
\end{gathered}
$$

3. It is not difficult to find symmetries of the "second order" using essentially the same considerations. By the "second order" symmetries we mean ones generating 
functions which depend only on $x, y, u, v, u_{(1)}, v_{(1)}, u_{(2)}, v_{(2)}$. The result is

$$
\begin{aligned}
\Phi= & \left(a y^{2}+b y+c\right) u_{(2)}-\frac{1}{2} \cdot\left(a x^{2}+d x+m\right) v_{(1)} \\
& +(2 a y+b+2 \alpha y-2 \gamma) u_{(1)}+\left[\left(-\frac{1}{2} \cdot a y-\frac{1}{4} \cdot b\right) x-\frac{1}{4} d y+2 \alpha+\delta\right] u+(\alpha x+\beta) v+h_{1}(x, y), \\
\Psi= & \left(a x^{2}+d x+m\right) v_{(2)}+(2 a x+d-2 \alpha x-2 \beta) v_{(1)} \\
& \quad-\frac{1}{2} \cdot\left(a y^{2}+b y+c\right) u_{(1)}+(-\alpha y+\gamma) u+\left[-\left(\frac{1}{2} \cdot a x+\frac{1}{4} d\right) y-\frac{1}{4} b x+\delta\right] v+h_{2}(x, y),
\end{aligned}
$$

where $a, b, c, d, m, \alpha, \beta, \gamma, \delta$ are constants and $\left(h_{1}, h_{2}\right)$ is an arbitrary solution of (1.5).

This experimental calculation forces us to suppose the existence of higher symmetries of arbitrary order.

4. Now we will show that this is indeed the case.

Let $f \in \mathscr{F}_{n}$. Then either $\left(\partial \phi / \partial u_{(n)}\right) \neq 0$ or $\left(\partial \Psi / \partial v_{(n)}\right) \neq 0$ and $l_{F}(f)$ is a first order polynomial with respect to variables $u_{(n+1)}, v_{(n+1)}$. Corresponding coefficients are $\left(\partial \bar{\psi} / \partial u_{(n)}\right),\left(\partial \phi / \partial v_{(n)}\right)$ and therefore they are to be zero. Since $\bar{\psi}=-2 \bar{D}_{x} \bar{\phi}, \bar{\phi}=-2 \bar{D}_{y} \bar{\psi}$ this shows that

$$
\bar{\phi}=A_{1} u_{(n)}+B_{1}, \quad \bar{\psi}=A_{2} v_{(n)}+B_{2},
$$

where $A_{i}, B_{i} \in \mathscr{F}_{n-1}$. From the latter one can conclude that $l_{F}(f)$ is a first order polynomial in variables $u_{(n)}, v_{(n)}$ of which some coefficients are $\bar{D}_{x} A_{1}, \bar{D}_{y} A_{2}$. Therefore

$$
\bar{D}_{x} A_{1}=0, \quad \bar{D}_{y} A_{2}=0
$$

and $A_{1}=A_{1}(y) ; A_{2}=A_{2}(x)$. Consideration of other coefficients leads to

$$
\begin{aligned}
& B_{1}=-\frac{1}{2} \cdot A_{2} v_{(n-1)}+C_{1} u_{(n-1)}+D_{1}, \\
& B_{2}=-\frac{1}{2} \cdot A_{1} u_{(n-1)}+C_{2} v_{(n-1)}+D_{2},
\end{aligned}
$$

where $C_{i}, D_{i} \in \mathscr{F}_{n-2}$.

Taking it into account one can see that $T_{F}(\bar{f})$ is a first order polynomial in $u_{(n-1)}$, $v_{(n-1)}$. As above its coefficients are to be zero. This gives the following equations:

$$
\begin{gathered}
\bar{D}_{x} C_{1}=\bar{D}_{y} C_{2}=0, \\
\frac{1}{2} \cdot C_{2}+\frac{\partial D_{1}}{\partial v_{(n-2)}}-\frac{1}{2} \cdot A_{2}^{\prime}(x)=0, \quad \frac{1}{2} \cdot C_{1}-\frac{1}{2} \cdot A_{1}^{\prime}(y)+\frac{\partial D_{2}}{\partial u_{(n-2)}}=0 .
\end{gathered}
$$

From these it is easy to find that $C_{1}=C_{1}(y), C_{2}=C_{2}(x)$ and also

$$
D_{1}=\frac{1}{2}\left(A_{2}^{\prime}(x)-C_{2}(x)\right) v_{(n-2)}+\alpha(x, y) u_{(n-2)}+F_{1},
$$




$$
D_{2}=\frac{1}{2}\left(A_{1}^{\prime}(y)-C_{1}(y)\right) u_{(n-2)}+\beta(x, y) v_{(n-2)}+F_{2},
$$

where $F_{1}, F_{2} \in \mathscr{F}_{n-3}$.

Substituting these expressions in $I_{F}(f)$ one can see that $l_{F}(J)$ is a first order polynomial in $u_{(n-2)}, v_{(n-2)}$. Its coefficients give the equations:

$$
\frac{\partial \alpha}{\partial x}+\frac{1}{4} \cdot A_{1}^{\prime}(y)=0, \quad \frac{\partial \beta}{\partial y}+\frac{1}{4} \cdot A_{2}^{\prime}(x)=0,
$$

solutions of which are

$$
\alpha=-\frac{1}{4} \cdot A_{1}^{\prime}(y) x+a(y), \quad \beta=-\frac{1}{4} \cdot A_{2}^{\prime}(x) y+b(x),
$$

where $a, b$ arbitrary functions.

Finally, we have

$$
\begin{aligned}
\Phi= & A_{1}(y) u_{(n)}-\frac{1}{2} \cdot A_{2}(x) v_{(n-1)}+C_{1}(y) u_{(n-1)}+\frac{1}{2} \cdot\left(A_{2}^{\prime}(x)-C_{2}(x)\right) v_{(n-2)} \\
& +\left(a_{1}(y)-\frac{1}{4} \cdot x A_{1}^{\prime}(y)\right) u_{(n-2)}+F_{1}, \\
\Psi= & A_{2}(x) v_{(n)}-\frac{1}{2} \cdot A_{1}(y) u_{(n-1)}+C_{2}(x) v_{(n-1)}+\frac{1}{2} \cdot\left(A_{1}^{\prime}(y)-C_{1}(y)\right) u_{(n-2)} \\
& +\left(a_{2}(x)-\frac{1}{4} \cdot y A_{2}^{\prime}(x)\right) v_{(n-2)}+F_{2},
\end{aligned}
$$

where $F_{1}, F_{2} \in \mathscr{F}_{n-3}$.

Our further considerations will be based on the next lemma.

Lemma. The operator

$$
\square=\left(\begin{array}{cc}
(a y+b) D_{x}+(-a y++d) D_{y}+\gamma-\frac{1}{2} \cdot a, & -\frac{1}{2} \cdot(a x+c-a y-b) \\
-\frac{1}{2} \cdot(a x+c-a y-b), \quad(a x+c) D_{x}+(-a x+d+b-c) D_{y}+\frac{1}{2} \cdot a-\gamma
\end{array}\right),
$$

where $a, b, c, d, \gamma$ are arbitrary constants commutes with $l_{F}$. In particular, $\square(f) \in \operatorname{sym} \mathscr{Y}$ if $f \in \operatorname{sym} \mathscr{Y}$.

Direct calculation proves it easily.

Now we will show by induction that coefficients $A_{1}, A_{2}$ in (2.4) are polynomials of order $\leqq n$ in which higher order coefficients coincide up to the sign $(-1)^{n-1}$.

The cases $n=0,1,2$ considered above give the beginning of the induction process. Supposing the induction hypothesis for $n=k$ let us consider a generating function of the 
form

$$
f=\left(\begin{array}{l}
\phi \\
\psi
\end{array}\right)=\left(\begin{array}{c}
A_{1}(y) u_{(k+1)}-\frac{1}{2} \cdot A_{2}(x) v_{(k)}+C_{1}(y) u_{(k)} \\
+\frac{1}{2} \cdot\left(A_{2}^{\prime}(x)-C_{2}(x)\right) v_{(k-1)}+\cdots+F_{1}, \\
A_{2}(x) v_{(k+1)}-\frac{1}{2} \cdot A_{1}(y) u_{(k)}+C_{2}(x) v_{(k)} \\
+\frac{1}{2} \cdot\left(A_{1}^{\prime}(y)-C_{1}(y)\right) u_{(k-1)}+\cdots+F_{2}
\end{array}\right)
$$

If $f_{1}^{0} \in \operatorname{sym} \mathscr{Y}$, then $\left\{f, f_{1}^{0}\right\} \in \operatorname{sym} \mathscr{Y}$ and also in view of the lemma $\square\left(\left\{f, f_{1}^{0}\right\}\right) \in \operatorname{sym} \mathscr{Y}$. It is not difficult to see, that

$$
\left\{\tilde{f}, f_{1}^{0}\right\}=\left(\begin{array}{c}
\frac{\partial \Phi}{\partial y} \\
\frac{\partial \Psi}{\partial y}
\end{array}\right)
$$

Choosing the constants entering into the operator $\square$ to $b e b=c=1, a=d=\gamma=0$ one can see that

$$
\square\left(\left\{f, f_{1}^{0}\right\}\right)=\left(\begin{array}{l}
\frac{1}{4} \cdot A_{1}^{\prime}(y) u_{(k)}+E_{1} \\
-\frac{1}{4} A_{2}^{\prime}(x) v_{(k)}+E_{2}
\end{array}\right),
$$

where $E_{1}, E_{2} \in \mathscr{F}_{k-1}$. By the induction hypothesis $A_{1}^{\prime}$ and $A_{2}^{\prime}$ are polynomials of order $k$ higher coefficients of which differ by the multiplier $(-1)^{k-1}$. This finishes the proof. Now we see that independent symmetries belonging to $\mathscr{F}_{n} \backslash \mathscr{F}_{n-1}$ must have the form

$$
\begin{gathered}
S_{n}=\left(\begin{array}{c}
y^{n} u_{(n)}+E_{1} \\
(-1)^{n-1} x^{n} v_{(n)}+E_{2}
\end{array}\right), \\
f_{n}^{i}=\left(\begin{array}{c}
y^{i} u_{(n)}+E_{1}^{\prime} \\
E_{2}^{\prime}
\end{array}\right), \\
g_{n}^{i}=\left(\begin{array}{c}
E_{1}^{\prime \prime} \\
x^{i} v_{(n)}+E_{2}^{\prime \prime}
\end{array}\right),
\end{gathered}
$$

where $E_{1}^{(j)}, E_{2}^{(j)} \in \mathscr{F}_{n-1}, 0 \leqq i<n$.

Of course, functions $E_{i}^{())}$are not defined uniquely. To avoid this inexactitude we put

$$
f_{n}^{i}=\frac{1}{n(n-1) \ldots(n-i+1)}\left(\frac{\partial}{\partial y}\right)^{n-i}\left(S_{n}\right)
$$




$$
\begin{gathered}
=\frac{i !}{n !}\left\{\ldots\left\{S_{n}, f_{1}^{0}\right\}, \ldots, f_{1}^{0}\right\} \quad(n-i \text { times }) \\
g_{n}^{i}=\frac{i !}{n !}\left\{\ldots\left\{S_{n}, g_{1}^{0}\right\}, \ldots, g_{1}^{0}\right\} \quad(n-i \text { times }) \\
S_{n}=\square^{n}\left(S_{0}\right)
\end{gathered}
$$

where constants entering into the operator $\square$ from the above lemma are chosen to be $a=-1, b=c=d=\gamma=0$. Then it is not difficult to check that the elements $S_{n}, f_{n}^{i}, g_{n}^{i}$ so defined have their higher part as in (2.6). Elements $f_{n}^{i}, g_{n}^{i}$ may be also defined by the formulas

$$
\begin{aligned}
& f_{n}^{i}=\frac{(-1)^{n-i+1} i !}{n !} X_{f_{1}^{0}}^{-i}\left(S_{n}\right), \\
& g_{n}^{i}=\frac{(-1)^{n-i+1} i !}{n !} X_{g_{1}^{0}}^{n-i}\left(S_{n}\right) .
\end{aligned}
$$

Here the operator $X_{f}$ acts as $X_{f} g=\{f, g\}$.

Using the following notation

$$
\begin{gathered}
X=\left(\begin{array}{cc}
0 & -\frac{1}{2} \\
0 & D_{x}
\end{array}\right), \quad Y=\left(\begin{array}{cc}
D_{y} & 0 \\
-\frac{1}{2} & 0
\end{array}\right), \\
\square=\left(\begin{array}{cc}
y\left(D_{y}-D_{x}\right)+\frac{1}{2} & \frac{1}{2} \cdot(x-y) \\
\frac{1}{2} \cdot(x-y) & x\left(D_{y}-D_{x}\right)-\frac{1}{2}
\end{array}\right),
\end{gathered}
$$

we have

$$
\begin{gathered}
f_{n}^{i}=\left[Y,\left[Y, \ldots,\left[Y, \square^{n}\right] \ldots\right]\left(S_{0}\right)\right. \\
g_{n}^{i}=\left[X,\left[X, \ldots,\left[X, \square^{n}\right] \ldots\right]\left(S_{0}\right)\right.
\end{gathered}
$$

In order to find the higher Jacobi brackets of functions $S_{n}, f_{n}^{i}, g_{n}^{i}, H$ we need commutator formulae for operators $X, Y, \square$. Direct calculation shows that

$$
\begin{gathered}
{[X, Y]=A l_{F}, \quad X Y=\frac{1}{4} \cdot E+B l_{F},} \\
{[\square, X]=C l_{F}+X, \quad[Y, \square)=D l_{F}+Y,}
\end{gathered}
$$

where

$$
E=\left(\begin{array}{ll}
1 & 0 \\
0 & 1
\end{array}\right), \quad B=\left(\begin{array}{rr}
0 & 0 \\
-\frac{1}{2} & 0
\end{array}\right), \quad A=\left(\begin{array}{rr}
0 & \frac{1}{2} \\
-\frac{1}{2} & 0
\end{array}\right),
$$




$$
C=\left(\begin{array}{cc}
0 & \frac{1}{2} \cdot(x-y) \\
\frac{1}{2} \cdot(y-x) & -1
\end{array}\right), \quad D=\left(\begin{array}{cc}
-1 & \frac{1}{2} \cdot(x-y) \\
\frac{1}{2}(y-x) & 0
\end{array}\right)
$$

The restriction of (2.9) on the solution space of the equation $I_{E}(\bar{\phi})=0$ looks like

$$
\begin{aligned}
& {[\sigma, \tau]=0, \quad[\hat{\square}, \sigma]=\sigma,} \\
& {[\tau, \hat{\square}]=\tau, \quad \sigma \tau=\frac{1}{4} \cdot E,}
\end{aligned}
$$

where $\sigma, \tau, \hat{\square}$ are restrictions of the operators $X, Y, \square$ on this space.

Lemma. The following bracket formulae are valid:

$$
\begin{gathered}
\left\{f_{n}^{n-i}, f_{m}^{m-j}\right\}=\sum_{0 \leqq k<l \leqq m+n-1} A_{k, l} f_{l}^{k}, \quad\left\{S_{m}, S_{n}\right\}=0, \\
\left\{g_{n}^{n-i}, g_{m}^{m-j}\right\}=\sum_{0 \leqq k<l \leqq m+n-1} B_{k, l} g_{l}^{k}, \\
\left\{f_{n}^{n-i}, g_{m}^{m-j}\right\}=\left\{\begin{array}{l}
\sum_{0 \leqq k<l \leqq m+n-1} M_{k, l} f_{l}^{k}, \quad i>j, \\
\sum_{0 \leqq k<l \leqq m+n-1} N_{k} S_{k}, \quad i=j, \\
0 \leqq k<l \leqq m+n-1 \\
\left\{S_{m}, H\right\}=\square^{m}(H), \quad\left\{f_{n}^{i}, H\right\}=l_{f_{n}^{i}} H, \\
\left\{g_{n}^{i}, H\right\}=l_{g_{n}^{i}} H,
\end{array}\right.
\end{gathered}
$$

where $A_{k, l}, B_{k, l}, M_{k, l}, N_{k}, L_{k, l}$ are some constants.

Proof. It is straightforwardly to deduce from (2.8) that

$$
f_{n}^{n-i}=\left(\sum_{j=0}^{i} C_{n}^{j}(-1)^{j} \tau^{i-j} \hat{\square}^{n} \tau^{j}\right) S_{0} .
$$

Also it is easy to see that

$$
\hat{\square}^{l} \tau^{n}=\tau^{n}(\hat{\square}-n)^{l} .
$$

Taking into consideration (2.13) one may rewrite (2.12) as

$$
f_{n}^{n-i}=\left(\tau^{i} \sum_{j=0}^{i}(-1)^{j} C_{n}^{j}(\hat{\square}-j)^{n}\right) S_{0}
$$


Next, it follows from (2.13) and (2.14) that

$$
\begin{aligned}
\left\{f_{m}^{m-l}, f_{n}^{n-i}\right\}= & {\left[\tau^{l} \sum_{k=0}^{l} C_{m}^{k}(-1)^{k}(\hat{\square}-k)^{m}, \tau^{i} \sum_{j=0}^{i}(-1)^{j} C_{n}^{j}(\hat{\square}-j)^{n}\right] S_{0} } \\
= & \tau^{l+i}\left\{\sum_{k=0}^{l}(-1)^{k} C_{m}^{k}(\hat{\square}-k-i)^{m}\left(\sum_{j=0}^{i}(-1)^{j} C_{m}^{j}(\hat{\square}-j)^{n}\right)\right. \\
& \left.-\sum_{j=0}^{i}(-1)^{j} C_{n}^{j}(\hat{\square}-j-l)^{n}\left(\sum_{k=0}^{l}(-1)^{k} C_{m}^{k}(\hat{\square}-k)^{m}\right)\right\} S_{0} .
\end{aligned}
$$

The formula

$$
\left[\tau, \ldots,\left[\tau, \hat{\square}^{i+k}\right] \ldots\right]=\sum_{j=0}^{k} c_{j} \tau^{i} \hat{\square}^{j} \quad(i \text { times })
$$

in which $c_{j}$ are some constants gives the possibility to express operators $\tau^{i} \hat{\square}^{l}$ as linear combinations of commutators of the form $\left[\tau, \ldots,\left[\tau, \hat{\square}^{p}\right] \ldots\right]$. This remark together with (2.15) leads to the equality

$$
\left\{f_{n}^{n-i}, f_{m}^{m-l}\right\}=\sum_{0 \leqq j \leqq k \leqq m+n-1} A_{j, k} f_{k}^{j}
$$

in which $A_{j, k}$ are some constants.

The second of the equalities (2.11) is proved similarly as is the third. In the last case one needs to use additionally the equality $\sigma \tau=\frac{1}{4} E$. Other brackets in (2.11) are obtained by direct calculations.

Remark. Let $\mathscr{G}$ be the 3-dimensional Lie algebra generated by elements $\tau, \sigma$, $\hat{\square}$ with respect to the usual commutator as the Lie operation. Elements $\sigma^{i} \tau^{j} \hat{\square}^{n}$ constitute a basic of its enveloping algebra $U(\mathscr{G})$. It is not difficult to see that the Lie algebra

$$
A(\mathscr{G})=U(\mathscr{G}) / \mathscr{T},
$$

where the ideal $\mathscr{T}$ is generated by the element $\sigma \tau-\frac{1}{4} E$ is isomorphic to the Lie algebra sym $\mathscr{Y}, \mathscr{Y}$ being (1.5). This shows that elements

$$
R_{n}^{i}=\sigma^{i} \hat{\square}^{n}\left(S_{0}\right), \quad K_{n}^{i}=\tau^{i} \hat{\square}^{n}\left(S_{0}\right) \quad 0 \leqq i \leqq n
$$

and some of $H$ 's constitute a basis of sym $\mathscr{Y}$. Jacobi brackets in this basis looks as follows: 


$$
\begin{gathered}
\left\{R_{n}^{i}, K_{m}^{j}\right\}=\left\{\begin{array}{l}
\sum_{k=1}^{n}(-i)^{k} C_{n}^{k} R_{m+k}^{0}-\sum_{l=1}^{m}(-i)^{l} C_{m}^{l} K_{n+l}^{0}, \quad i=j \\
\sum_{k=1}^{n}(-i)^{k} C_{n}^{k} R_{m+k}^{i-j}-\sum_{l=1}^{m}(-j)^{l} C_{m}^{l} R_{n+l}^{i-j}, \quad i>j \\
\sum_{k=1}^{n}(-i)^{k} C_{n}^{k} K_{m+k}^{j-i}-\sum_{l=1}^{m}(-j)^{l} C_{m}^{l} K_{n+l}^{j-i}, \quad j>i
\end{array}\right. \\
\left\{R_{n}^{i}, H\right\}=\sigma^{i} \hat{\square}^{n}(H), \quad\left\{K_{n}^{i}, H\right\}=\tau^{i} \hat{\square}^{n}(H) .
\end{gathered}
$$

Now summarizing all the above we have:

Theorem 1. The algebra of higher symmetries of the equation (1.5) is generated by elements $S_{n}, f_{n}^{i}, g_{n}^{i}$ and $H, 0 \leqq i \leqq n$, as a linear space, and by elements $S_{n}, f_{1}^{0}, g_{1}^{0}, H$ as $a$ Lie algebra. Moreover the Lie operation in it is described by formulae (2.11) or by formulae (2.16).

\section{Conservation laws of equations (1.5)}

Let us remember that generating functions of conservation laws are contained in $\operatorname{ker} T_{F}^{*}$. Therefore our first step is to solve the equation (see Section 0)

$$
T_{F}^{*} f=0,
$$

where

$$
f=\left(\begin{array}{l}
\Phi \\
\psi
\end{array}\right), \quad I_{F}^{*}=\left(\begin{array}{cc}
-\bar{D}_{x} & \frac{1}{2} \\
\frac{1}{2} & -\bar{D}_{y}
\end{array}\right)
$$

1. If the generating function $f$ depends on $x, y, u, v, u_{x}, v_{x}, u_{y}, v_{y}$, then $f$ is a function only in the variables $x, y, u, v, u_{(1)}, v_{(1)}$. Performing calculations similar to ones at the beginning of Section 2, we obtain

$$
\begin{aligned}
& \bar{\phi}=(\alpha y+\beta) u_{(1)}+\frac{1}{2} \cdot(\alpha x+\gamma) v+\left(\delta-\frac{1}{2} \cdot \alpha\right) u+B_{1}(x, y), \\
& \psi=(\alpha x+\gamma) v_{(1)}+\frac{1}{2} \cdot(\alpha y+\beta) u+\left(\frac{1}{2} \cdot \alpha-\delta\right) v+B_{2}(x, y),
\end{aligned}
$$

where $\alpha, \beta, \gamma, \delta$ are arbitrary constants and $\left(B_{1}, B_{2}\right)$ is an arbitrary solution of the system

$$
\frac{\partial B_{1}}{\partial x}-\frac{1}{2} B_{2}=0, \quad \frac{\partial B_{2}}{\partial y}-\frac{1}{2} B_{1}=0
$$


2. Suppose $f$ to be a solution of (3.1) such that $f \in \mathscr{F}_{n}$ and $\left(\partial \phi / \partial u_{(n)}\right) \neq 0$ or $\left(\partial \psi / \partial v_{(n)}\right) \neq 0$. Word by word repetition of arguments as used in Section 2.3 shows that

$$
\begin{aligned}
\Phi= & A_{1}(y) u_{(n)}+\frac{1}{2} A_{2}(x) v_{(n-1)}+C_{1}(y) u_{(n-1)}+\frac{1}{2}\left(C_{2}(x)-A_{2}^{\prime}(x)\right) v_{(n-2)} \\
& +\left(a_{1}(y)-\frac{1}{4} A_{1}^{\prime}(y) x\right) u_{(n-2)}+F_{1}, \\
\Psi= & A_{2}(x) v_{(n)}+\frac{1}{2} A_{1}(y) u_{(n-1)}+C_{2}(x) v_{(n-1)}+\frac{1}{2}\left(C_{1}(y)-A_{1}^{\prime}(y)\right) u_{(n-2)} \\
& +\left(a_{2}(x)-\frac{1}{4} \cdot y A_{2}^{\prime}(x)\right) v_{(n-2)}+F_{2},
\end{aligned}
$$

where $F_{1}, F_{2} \in \mathscr{F}_{n-3}$.

As above coefficients $A_{1}$ and $A_{2}$ in (3.4) are polynomials of order $n$, in which higher order coefficients differ by the multiplier $(-1)^{n}$. To prove this we will use the formula (0.7) with

$$
\begin{gathered}
f=f_{1}^{0}=\left(\begin{array}{c}
u_{(1)} \\
-\frac{1}{2} u
\end{array}\right) \in \operatorname{sym} \mathscr{Y} \\
g=\left(\begin{array}{c}
A_{1}(y) u_{(n+1)}+\frac{1}{2} A_{2}(x) v_{(n)}+C_{1}(y) u_{(n)}+F_{1} \\
A_{2}(x) v_{(n+1)}+\frac{1}{2} A_{1}(y) u_{(n)}+C_{2}(x) v_{(n)}+F_{2}
\end{array}\right) .
\end{gathered}
$$

As the result we obtain a new generating function

$$
\hat{f}=\overline{l_{g}(f)}+\overline{\Delta^{*}(f)} \in \operatorname{ker} \overline{l_{F}^{*}} .
$$

If $\delta=\square^{*}$, where $\square$ is the operator $(2.5)$, then $l_{F}^{*} \circ \delta=\delta \circ l_{F}^{*}$. Therefore $\delta(h) \in \operatorname{ker} \overline{l_{F}^{*}}$ if $h \in \operatorname{ker} \bar{l}_{F}^{*}$. Specializing constants in $\delta$ to be $b=d=-1$ and the others to be zero we see that

$$
\delta(\hat{f})=\left(\begin{array}{c}
-\frac{1}{4} A_{1}^{\prime}(y) u_{(n)}+F_{1} \\
\frac{1}{4} A_{2}^{\prime}(x) v_{(n)}+F_{2}
\end{array}\right) \in \operatorname{ker} \overline{l_{F}^{*}}
$$

Now the polynomial property is proved by the same arguments as in Section 2. Also independent generating functions of conservation laws belonging to $\mathscr{F}_{n} \backslash \mathscr{F}_{n-1}$ may be chosen in the form

$$
\begin{gathered}
T_{n}=\left(\begin{array}{c}
y^{n} u_{(n)}+F_{1} \\
(-1)^{n} x^{n} v_{(n)}+F_{2}
\end{array}\right), \\
P_{n}^{i}=\left(\begin{array}{c}
y^{i} u_{(n)}+F_{1}^{\prime} \\
F_{2}^{\prime}
\end{array}\right), \\
Q_{n}^{i}=\left(\begin{array}{c}
F_{1}^{\prime \prime} \\
x^{i} v_{(n)}+F_{2}^{\prime \prime}
\end{array}\right),
\end{gathered}
$$

where $F_{j}^{(i)} \in \mathscr{F}_{n-1}$. 
Using the formula (0.7) it is not difficult to check that expressions

$$
\begin{gathered}
2 P_{2 k}^{i}=f_{2 k}^{i}\left[T_{0}\right], \quad 2 Q_{2 k}^{i}=g_{2 k}^{i}\left[T_{0}\right], \\
2 P_{2 k+1}^{i}=f_{2 k}^{i}\left[P_{1}^{0}\right], \quad 2 Q_{2 k+1}^{i}=g_{2 k}^{i}\left[Q_{1}^{0}\right], \\
T_{n}=\delta^{n}\left(T_{0}\right),
\end{gathered}
$$

where

$$
P_{1}^{0}=\left(\begin{array}{c}
u_{(1)} \\
\frac{1}{2} \cdot u
\end{array}\right), \quad Q_{1}^{0}=\left(\begin{array}{c}
\frac{1}{2} \cdot v \\
v_{(1)}
\end{array}\right), \quad T_{0}=\left(\begin{array}{c}
u \\
-v
\end{array}\right)
$$

are the form (3.5). For this reason we use (3.6) as an exact definition of functions $T_{n}$, $P_{n}^{i}$, $Q_{n}^{i}$. These functions together with functions (3.3) generate all solutions of (3.1).

In order for a solution of (3.1) to be the generating function of a conservation law the representation (0.6) should take place. The function $T_{0}$ satisfies this condition. In fact,

$$
l_{T_{0}}=\left(\begin{array}{rr}
1 & 0 \\
0 & -1
\end{array}\right), \quad l_{F}^{*}\left(T_{0}\right)=\left(\begin{array}{rr}
-1 & 0 \\
0 & 1
\end{array}\right)\left(\begin{array}{cc}
u_{x} & +\frac{1}{2} \cdot v \\
\frac{1}{2} \cdot u & +v_{y}
\end{array}\right)
$$

Therefore

$$
A=\left(\begin{array}{rr}
-1 & 0 \\
0 & 1
\end{array}\right)
$$

and $l_{T_{0}}+A^{*}=0$. This fact and formulae (3.6) show that functions $P_{2 k}^{i}$ and $Q_{2 k}^{i}$ are generating functions of some conservation laws.

On the contrary we shall prove that no linear combination of functions $P_{n}^{i}, Q_{n}^{i}, T_{n}$, $n=2 k+1$, is the generating function of a conservation law of $\mathscr{Y}$.

First, remark that $P_{n}^{i}$ (respectively, $Q_{n}^{i}$ ) owing to (3.5) may be presented as a linear combinations of derivatives $\left(\partial^{s} T_{n} / \partial y^{s}\right)$ (respectively, $\left.\left(\partial^{s} T_{n} / \partial x^{s}\right)\right), 0 \leqq s \leqq k$. Therefore it suffices to prove that for the function

$$
\phi=\sum_{k} \alpha_{2 k+1} T_{2 k+1}+\sum_{0 \leqq i \leqq 2 k+1}\left(\beta_{k, i} \frac{\partial^{i} T_{2 k+1}}{\partial y^{i}}+\gamma_{k, i} \frac{\partial^{i} T_{2 k+1}}{\partial x^{i}}\right),
$$

where $\alpha_{n}, \beta_{k, i}, \gamma_{k, i}$ are arbitrary constants, no representation of the form (0.6) exists.

The left hand side of $(0.6)$ adopted for a function $\phi$ we will denote $H(\phi)$. Also the operator $A$ entering in it will be denoted $B(\phi)$. With this notation we have

$$
H(\phi)=l_{\phi}+B(\phi)^{*} .
$$

It is easy to see that $H\left(\lambda_{i} \phi_{i}\right)=\sum \lambda_{i} H\left(\phi_{i}\right)$, if $\lambda_{i}$ are some constants. For this reason we need some explicit formulae for operators $l_{\psi}$ and $B(\psi)$, where

$$
\psi=\frac{\partial^{i} T_{n}}{\partial x^{i}} \text { or } \frac{\partial^{i} T_{n}}{\partial y^{i}}
$$


First, supposing operator $\delta$ and matrix $A$ to be as above we see that

$$
l_{T_{n}}=l_{\delta_{n} T_{0}}=\delta^{n} l_{T_{0}}=-\delta^{n} A .
$$

Further, using the following directly verifiable formula

$$
l_{(\partial B / \partial x)}=\left[\frac{\partial}{\partial x}, l_{B}\right]
$$

we see, that

$$
l_{\left(\partial t T_{n} / \partial x^{\prime}\right)}=-\left[\frac{\partial}{\partial x}, \ldots,\left[\frac{\partial}{\partial x}, \delta^{n} A\right] \ldots\right] \quad(i \text { times }) .
$$

Now it follows from $\delta \circ l_{F}^{*}=l_{F}^{*} \circ \delta$, that $l_{F}^{*}\left(T_{n}\right)=l_{F}^{*}\left(\delta^{n} T_{0}\right)=\delta^{n} l_{F}^{*}\left(T_{0}\right)=\left(\delta^{n} A\right)(F)$ and we see that $B\left(T_{n}\right)=\delta^{n} A$. But

$$
\frac{\partial}{\partial x} l_{F}^{*}=l_{F}^{*} \frac{\partial}{\partial x} \text { and } \frac{\partial F}{\partial x}=0
$$

Therefore

$$
l_{F}^{*}\left(\frac{\partial^{i} T_{n}}{\partial x^{i}}\right)=\frac{\partial^{i}}{\partial x^{i}}\left(l_{F}^{*}\left(T_{n}\right)\right)=\frac{\partial^{i}}{\partial x^{i}}\left(\left(\delta^{n} A\right)(F)\right)=\left[\frac{\partial}{\partial x}, \ldots,\left[\frac{\partial}{\partial x}, \delta^{n} A\right] \ldots\right](F)
$$

and, finally

$$
H\left(\frac{\partial^{i} T_{n}}{\partial x^{i}}\right)=\left[\frac{\partial}{\partial x}, \ldots,\left[\frac{\partial}{\partial x}, A \delta^{* n}-\delta^{n} A\right] \ldots\right] \text { (i times). }
$$

Obviously a similar formula for $H\left(\partial^{i} T_{n} / \partial y^{i}\right)$ is valid. For any odd $n$ direct calculations lead to the formula

$$
\left(A \delta^{* n}-\delta^{n} A\right)\left(\begin{array}{l}
u \\
v
\end{array}\right)=\left(\begin{array}{c}
2 y^{n} u_{(n)}+E_{1} \\
-2 x^{n} v_{(n)}+E_{2}
\end{array}\right)
$$

where the $E_{i}$ are polynomials of order $<n$ in $x, y$ in which coefficients are linear polynomials in variables $u_{(n)}$ and $v_{(n)}$ with coefficients belonging to $\mathscr{F}_{n-1}$. Now in spite of (3.7) and (3.8) one can conclude that

$$
\bar{H}(\phi)\left(\begin{array}{l}
u \\
v
\end{array}\right)=\sum_{k}\left[\alpha_{k}+\sum_{0 \leqq i \leqq 2 k+1}\left(\beta_{k, i} \frac{\partial^{i}}{\partial y^{i}}+\gamma_{k, i} \frac{\partial^{i}}{\partial x^{i}}\right)\right]\left(g_{k}\right),
$$

where $g_{k}$ is the right hand side of (3.8) taken for $n=2 k+1$. If not all of the numbers $\alpha_{k}$, $\beta_{k, i}, \gamma_{k, i}$ are equal to zero, i.e. if $\phi \neq 0$, then the right hand side of the latter equality is not zero. This is clear from the form of $g_{k}$. But

$$
\left(\bar{B} \circ I_{F}\right)\left(\begin{array}{l}
u \\
v
\end{array}\right)=\bar{B}\left(I_{F}\left(\begin{array}{l}
u \\
v
\end{array}\right)\right)=\bar{B}(\bar{F})=0 .
$$


Therefore the equality $\bar{H}(\phi)=\bar{B} \circ T_{F}$ is impossible. Finally, putting all above together we obtain:

Theorem 2. The set of generating functions for the conservation laws of equation (1.5) is generated as a vector space by solutions of (3.3) and by elements $T_{2 n}, P_{2 n}^{i}, Q_{2 n}^{i}$. Moreover, the following formulae hold

$$
\begin{array}{ll}
2 P_{2 n}^{i}=l_{T_{0}}\left(f_{2 n}^{i}\right)+\Delta_{n, i}^{*}\left(T_{0}\right) & 0 \leqq i \leqq 2 n, \\
2 Q_{2 n}^{i}=l_{T_{0}}\left(g_{2 n}^{i}\right)+\nabla_{n, i}^{*}\left(T_{0}\right) & 0 \leqq i \leqq 2 n,
\end{array}
$$

where operators $\Delta_{n, i}, \nabla_{n, i}$ are defined by the equation

$$
l_{F}\left(f_{2 n}^{i}\right)=\Delta_{n, i}(F), \quad l_{F}\left(g_{2 n}^{i}\right)=\nabla_{n, i}(F) .
$$

It is straightforward that a conserved current corresponding to the generating function $(\phi, \psi)$ is $(u \phi,-v \psi)$ (see Section 0.6). In other words for an arbitrary closed curve $\Gamma$ in the $(x, y)$-plane the equality

$$
\int_{\Gamma}(u \phi d y-v \psi d x)=0
$$

takes place supposing $(u, v)$ is a solution of $(1.5)$.

\section{Higher symmetries and conservation laws of the plasticity equations}

In Section 2 and Section 3 there were found generating functions of symmetries and conservation laws for equations (1.5). These are expressed in the variables $\xi, \eta, \bar{x}, \bar{y}$. Below we will transform them into ones for the equation (1.1).

1. The Cartan forms corresponding to the coordinate systems $(\xi, \eta, \bar{x}, \bar{y})$ and $(x, y, \sigma, \theta)$ have the form

$$
\begin{aligned}
& U_{1}^{\prime}=d \bar{x}-\bar{x}_{\xi} d \xi-\bar{x}_{\eta} d \eta, \\
& U_{2}^{\prime}=d \bar{y}-\bar{y}_{\xi} d \xi-\bar{y}_{\eta} d \eta,
\end{aligned}
$$

and

$$
\begin{aligned}
& U_{1}=d \sigma-\sigma_{x} d x-\sigma_{y} d y, \\
& U_{2}=d \theta-\theta_{x} d x-\theta_{y} d y,
\end{aligned}
$$

respectively.

It follows from the general theory [3] that

$$
\left(\begin{array}{l}
U_{1} \\
U_{2}
\end{array}\right)=\lambda\left(\begin{array}{l}
U_{1}^{\prime} \\
U_{2}^{\prime}
\end{array}\right)
$$


where $\lambda$ is a $2 \times 2$-matrix. It is not difficult to see that

$$
\lambda=\left(\begin{array}{ll}
-\sigma_{x} \cos \theta-\sigma_{y} \sin \theta & \sigma_{x} \sin \theta-\sigma_{y} \cos \theta \\
-\theta_{x} \cos \theta-\theta_{y} \sin \theta & \theta_{x} \sin \theta-\theta_{y} \cos \theta
\end{array}\right)
$$

It follows that symmetry generating functions for the equations (1.5), say $f^{\prime}$, and ones for the equation (1.1), say $f$, are related by the formula (see [3])

$$
f=\lambda f^{\prime} .
$$

2. It follows from (4.5) that generating functions $H_{1}, \ldots, H_{5}$ of classical infinitesimal symmetries are

$$
\begin{aligned}
& H_{1}=\left(\begin{array}{c}
-\cos \frac{1}{2}(\eta-\xi) \\
\sin \frac{1}{2}(\eta-\xi)
\end{array}\right), \quad H_{2}=\left(\begin{array}{c}
\sin \frac{1}{2}(\eta-\xi) \\
-\cos \frac{1}{2}(\eta-\xi)
\end{array}\right), \\
& H_{3}=S_{0}, \quad H_{4}=\frac{1}{2}\left(g_{1}^{0}-f_{1}^{0}\right), \quad H_{5}=\frac{1}{2 k}\left(g_{1}^{0}+f_{1}^{0}\right) .
\end{aligned}
$$

Functions $K=\lambda H$ where $H=\left(h_{1}, h_{2}\right)$ are arbitrary solutions of (1.5) as well as the function

$$
H_{6}=\left(\begin{array}{c}
-2 k \theta+\sigma_{x} \xi_{1}+\sigma_{y} \xi_{2} \\
-\frac{\sigma}{k}+\theta_{x} \xi_{1}+\theta_{y} \xi_{2}
\end{array}\right)
$$

where

$$
\begin{gathered}
\xi_{1}=-x \cos 2 \theta-y \sin 2 \theta-y \frac{\sigma}{k} \\
\xi_{2}=y \cos 2 \theta-x \sin 2 \theta+x \frac{\sigma}{k}
\end{gathered}
$$

corresponding to the function $S_{1}$ in $(\xi, \eta, \bar{x}, \bar{y})$-coordinates correspond to the new previously unknown classical symmetries of the equation (1.1) (see [1]). It shows that the equation (1.1) has the infinite-dimensional algebra $L^{\infty}$ of classical infinitesimal symmetries generated by operators:

$$
X_{1}, X_{2}, X_{3}, X_{4}, X_{5}, \quad X_{6}=\xi_{1} \frac{\partial}{\partial x}+\xi_{2} \frac{\partial}{\partial y}-2 k \theta \frac{\partial}{\partial \sigma}-\frac{\sigma}{k} \frac{\partial}{\partial \theta}, \quad X=\xi \frac{\partial}{\partial x}+\eta \frac{\partial}{\partial y},
$$


where $\xi_{1}, \xi_{2}$ are defined by $(4.6)$ and $(\xi, \eta)$ is an arbitrary solution of the following linear system of differential equations

$$
\begin{aligned}
& 2 k\left(\sin 2 \theta \frac{\partial \eta}{\partial \sigma}+\cos 2 \theta \frac{\partial \xi}{\partial \sigma}\right)-\frac{\partial \xi}{\partial \theta}=0 \\
& 2 k\left(\sin 2 \theta \frac{\partial \xi}{\partial \sigma}-\cos 2 \theta \frac{\partial \eta}{\partial \sigma}\right)-\frac{\partial \eta}{\partial \theta}=0
\end{aligned}
$$

Now we need to rewrite the recursion operator $\square(2.6)$ in terms of the initial coordinates. Obviously, a generating function of the form $\square f^{\prime}$ adopted to the coordinates $(\xi, \eta, \bar{x}, \bar{y})$ corresponds to a generating function $\tilde{\square} f$ adopted to the coordinates $(x, y, \sigma, \theta)$ if $f^{\prime}$ corresponds to $f$ and $\tilde{\square}=\lambda \square \lambda^{-1}$. In order to rewrite the operator $\square$ in terms of the initial coordinates we will express $D_{\xi}, D_{\eta}$ by means of $D_{x}, D_{y}$. It follows from the general theory that

$$
D_{\xi}=\lambda_{1} D_{x}+\lambda_{2} D_{y}, \quad D_{\eta}=\mu_{1} D_{x}+\mu_{l} D_{y}
$$

where $\lambda_{i}, \mu_{i}$ are functions on $J^{\infty}$.

Since $D_{\xi}(x)=\lambda_{1}, D_{\xi}(y)=\lambda_{2}$, etc. then

$$
\begin{aligned}
& D_{\xi}=D_{\xi}(x) D_{x}+D_{\xi}(y) D_{y}, \\
& D_{\eta}=D_{\eta}(x) D_{x}+D_{\eta}(y) D_{y},
\end{aligned}
$$

and therefore

$$
\begin{aligned}
& D_{\xi}=\frac{k}{\sigma_{x} \theta_{y}-\sigma_{y} \theta_{x}}\left(\left(\frac{\sigma_{y}}{2 k}+\theta_{y}\right) D_{x}-\left(\frac{\sigma_{x}}{2 k}+\theta_{x}\right) D_{y}\right), \\
& D_{\eta}=\frac{k}{\sigma_{x} \theta_{y}-\sigma_{y} \theta_{x}}\left(\left(\theta_{y}-\frac{\sigma_{y}}{2 k}\right) D_{x}+\left(\frac{\sigma_{x}}{2 k}-\theta_{x}\right) D_{y}\right) .
\end{aligned}
$$

Taking $a=-1$ and the other constants in $\square$ to be zero we obtain

$$
\begin{gathered}
\tilde{\square}=\left\|b_{i j}\right\| \quad i, j=1,2 \\
b_{11}=\frac{\sigma}{k} E+2 k I^{-1} \theta\left[\theta_{x}^{2}+\theta_{y}^{2}+C E(D)-D E(C)\right]-I^{-1} E(I), \\
b_{12}=8 k^{2} \theta E+2 k I^{-1}\left[\cos 2 \theta\left(\theta_{x}^{2}-\theta_{y}^{2}\right)-2 \theta_{x} \theta_{y} \sin 2 \theta-E(I)\right]+4 k[C E(D)-D E(C)], \\
b_{21}=\frac{\theta}{4 k} E+\theta I^{-1}\left[C E(D)-D E(C)+E(I)+C^{2}-D^{2}\right]+\frac{1}{4 k}, \\
b_{22}=\frac{\sigma}{k} E+2 k \theta I^{-1}\left[C E(D)-D E(C)-\theta_{x}^{2}-\theta_{y}^{2}\right]-I^{-1} E(I),
\end{gathered}
$$


where

$$
\begin{array}{cl}
C=-\theta_{x} \cos \theta-\theta_{y} \sin \theta, & D=\theta_{x} \sin \theta-\theta_{y} \cos \theta, \\
E=\sigma_{x} D_{y}-\sigma_{y} D_{x}, \quad I=\sigma_{x} \theta_{y}-\sigma_{y} \theta_{x} .
\end{array}
$$

4. Now we have all that is necessary to describe higher symmetries of the equations (1.1). Let

$$
Z_{0}=f_{3}=\left(\begin{array}{l}
-x \sigma_{x}-y \sigma_{y} \\
-x \theta_{x}-y \theta_{y}
\end{array}\right)
$$

and

$$
Z_{n}=\tilde{\square}^{n}\left(Z_{0}\right)
$$

where $\tilde{\square}$ is the operator (4.8).

Also, let

$$
\begin{gathered}
\phi_{1}^{0}=\left(\begin{array}{c}
k-\frac{1}{2}\left(-y \sigma_{x}+x \sigma_{y}\right) \\
-\frac{1}{2}-\frac{1}{2}\left(-y \theta_{x}+x \theta_{y}\right)
\end{array}\right)=\lambda g_{1}^{0}, \\
\psi_{1}^{0}=\left(\begin{array}{c}
k+\frac{1}{2}\left(-y \sigma_{x}+x \sigma_{y}\right) \\
\frac{1}{2}+\frac{1}{2}\left(-y \theta_{x}+x \theta_{y}\right)
\end{array}\right)=\lambda f_{1}^{0},
\end{gathered}
$$

and

$$
\begin{aligned}
& \phi_{n}^{i}=(-1)^{n+1-i} \frac{i !}{n !}\left\{\ldots\left\{Z_{n}, \phi_{1}^{0}\right\}, \ldots, \phi_{1}^{0}\right\} \quad(n-i \text { times }) \\
& \psi_{n}^{i}=(-1)^{n+1-i} \frac{i !}{n !}\left\{\ldots\left\{Z_{n}, \psi_{1}^{0}\right\}, \ldots, \psi_{1}^{0}\right\} \quad(n-i \text { times }) .
\end{aligned}
$$

Taking into consideration Theorem 1 we obtain:

Theorem 3. The algebra of higher symmetries of the system (1.1) is generated as a vector space by elements $K, Z_{n}, \phi_{n}^{i}, \psi_{n}^{i}, 0 \leqq i<n$, and as a Lie algebra by elements $K, Z_{n}$, $\phi_{1}^{0}, \psi_{1}^{0}$.

5. Now we are going to describe generating functions of conservation laws for equations (1.1).

Let $F$ (respectively, $F^{\prime}$ ) be the left hand side of equations (1.1) (respectively, of (1.5)) which are supposed to be written in coordinates $(x, y, \sigma, \theta)$. Then

$$
F=\Lambda F^{\prime}
$$


where $\Lambda$ is a $2 \times 2$-matrix. It is not difficult to find it directly:

$$
\Lambda=2\left(\sigma_{x} \theta_{y}-\sigma_{y} \theta_{x}\right)\left(\begin{array}{cc}
-\sin \theta & \cos \theta \\
\cos \theta & \sin \theta
\end{array}\right)
$$

Using general properties of universal linearization operators (see [3]) and the formula (4.10) we have

$$
l_{F}=\overline{l_{\Lambda F^{\prime}}}=\Lambda T_{F^{\prime}}+\bar{F}^{\prime} T_{\Lambda}=\Lambda T_{F^{\prime}}
$$

and

$$
\overline{l_{F}^{*}}=\overline{l_{F}^{*}} \circ \bar{\Lambda}^{*}
$$

It follows that solutions of equations $l_{F}^{*} f=0$ and $\overline{l_{F}^{*}} f^{\prime}=0$ are transformed into each other via transformations

$$
\bar{f}^{\prime}=\bar{\Lambda}^{*} \bar{f}, \quad \bar{f}=\left(\bar{\Lambda}^{*}\right)^{-1} \bar{f}^{\prime}
$$

This leads us to the formula

$$
\tilde{\square}=\left(\Lambda^{*}\right)^{-1} \delta \Lambda^{*}
$$

relating recursion operators for equation $\overline{l_{F}^{*}} f=0$ in the initial coordinates and ones in the coordinates $(\xi, \eta, \bar{x}, \bar{y})$. Taking $\delta=\square^{*}$ (see (2.5)) where $b=-1, d=1$ and the other constants in $\square$ are equal to zero and using (4.7) we obtain

$$
\tilde{\square}=\frac{1}{2 k I}\left(\begin{array}{cc}
a_{11} \sin \theta-a_{21} \cos \theta & a_{12} \sin \theta-a_{22} \cos \theta \\
-a_{11} \cos \theta-a_{21} \sin \theta & 0
\end{array}\right),
$$

where

$$
\begin{gathered}
a_{11}=2 \sin \theta A-2 k I \cos \theta+2 B \sin \theta-\frac{1}{2} \cos \theta, \\
a_{12}=-2 \cos \theta A+2 k I \sin \theta-2 B \cos \theta-\frac{1}{2} \sin \theta, \\
2 a_{21}=\sin \theta, \quad 2 a_{22}=-\cos \theta \\
A=\sigma_{y} D_{x}-\sigma_{x} D_{y}, \quad I B=\sigma_{x} I_{y}-\sigma_{y} I_{x}, \quad I=\sigma_{x} \theta_{y}-\sigma_{y} \theta_{x} .
\end{gathered}
$$

6. Now we are able to describe generating functions for conservation laws of the equations (1.1). Let

$$
Z_{0}=\left(\bar{\Lambda}^{*}\right)^{-1}\left(\begin{array}{c}
\bar{x} \\
\bar{y}
\end{array}\right)=\left(\begin{array}{c}
\frac{1}{2} y \\
-\frac{1}{2} x
\end{array}\right)
$$


and

$$
Z_{n}=\tilde{\square}^{n}\left(Z_{0}\right)
$$

Also, let

$$
\begin{aligned}
N_{2 n}^{i} & =2\left(\bar{\Lambda}^{*}\right)^{-1} P_{2 n}^{i}, \\
M_{2 n}^{i} & =2\left(\Lambda^{*}\right)^{-1} Q_{2 n}^{i}, \\
R & =\left(\Lambda^{*}\right)^{-1} B,
\end{aligned}
$$

where $B=\left(B_{1}, B_{2}\right)$ is a solution of (3.3). Then

$$
\begin{aligned}
& 2 N_{2 n}^{i}=\overline{l_{Z_{0}}\left(\psi_{2 n}^{i}\right)}+\overline{\Delta_{n, i}^{*}\left(Z_{0}\right)}, \\
& 2 M_{2 n}^{i}=\overline{l_{Z_{0}}\left(\phi_{2 n}^{i}\right)}+\overline{\nabla_{n, i}^{*}\left(Z_{0}\right)},
\end{aligned}
$$

where

$$
l_{F}\left(\psi_{2 n}^{i}\right)=\Delta_{n, i}(F), \quad l_{F}\left(\phi_{2 n}^{i}\right)=\nabla_{n, i}(F) .
$$

With this notation and in virtue of Theorem 2 we have:

Theorem 4. The group of generating functions corresponding to conservation laws of equation (1.1) is generated as the vector space by elements $Z_{2 n}, N_{2 n}^{i}, M_{2 n}^{i}, 0 \leqq i<2 n$, and $R$.

7. Finally, the "integral" form of the conservation laws of (1.1) corresponding to the generating functions $(\phi, \psi)$ in the initial coordinates is

$$
\begin{gathered}
\int_{\Gamma}\left(-\phi \bar{x} \cdot\left(\frac{\sigma_{x}}{2 k}+\theta_{x}\right)+\psi \bar{y} \cdot\left(\frac{\sigma_{x}}{2 k}-\theta_{x}\right)\right) d x+\left(\phi \bar{x} \cdot\left(\frac{\sigma_{y}}{2 k}+\theta_{y}\right)+\psi \bar{y} \cdot\left(\frac{\sigma_{y}}{2 k}-\theta_{y}\right)\right) d y=0, \\
\bar{x}=x \cos \theta+y \sin \theta, \quad \bar{y}=-x \sin \theta+y \cos \theta .
\end{gathered}
$$

Here $\Gamma$ is an arbitrary closed curve in the $(x, y)$-plane and $(\sigma, \theta)$ is a solution of $(1.1)$.

A conserved current corresponding to $T_{0}$ is $\bar{x}^{2} d \eta+\bar{y}^{2} d \xi$. Rewriting it in the initial coordinates we obtain the form

$$
\begin{aligned}
\omega= & {\left[2 x \theta_{x}(x \cos 2 \theta+y \sin 2 \theta)+\left(x^{2}+y^{2}\right) \theta_{y} \sin 2 \theta\right] d x } \\
& +\left[\left(x^{2}+y^{2}\right) \theta_{x} \sin \theta+2 y \theta_{y}(x \sin 2 \theta-y \cos 2 \theta)\right] d y .
\end{aligned}
$$

Therefore by the definitions currents $\ni_{\phi l_{n}}(\omega)$ and $\ni_{\psi \psi_{n}}(\omega)$ correspond to generating functions $M_{2 n}^{i}$ and $N_{2 n}^{i}$ respectively. 


\section{REFERENCES}

1. B. D. Annin, V. O. Bytev and S. I. Senashov, Group Properties of Elasticity and Plasticity Equations ("Nauka", Novosibirsk, 1985, in Russian).

2. N. H. Ibragimov, Group Transformations in the Mathematical Physics ("Nauka", Moscow, 1983, English translations: Reidel, 1985).

3. I. S. Krasilshchik, V. V. Lychagin and A. M. Vinogradov, Geometry of Jet Spaces and Nonlinear Partial Differential Equations (Gordon and Breach, New York, 1986).

4. L. V. Ovsiannikov, Group Analysis of Differential Equations ("Nauka", Moscow, 1978, English translation: Academic Press, 1982).

5. A. M. Vinogradov, Local symmetries and conservation laws, Acta Appl. Math. 2 (1984), 21-78.

Department of Mathematics

Krasnojarsky UniverstTy

660062 KRASNOJARSK, U.S.S.R.
Department of Mathematics MosCow UNIVERSITY

117234 Moscow, U.S.S.R. 